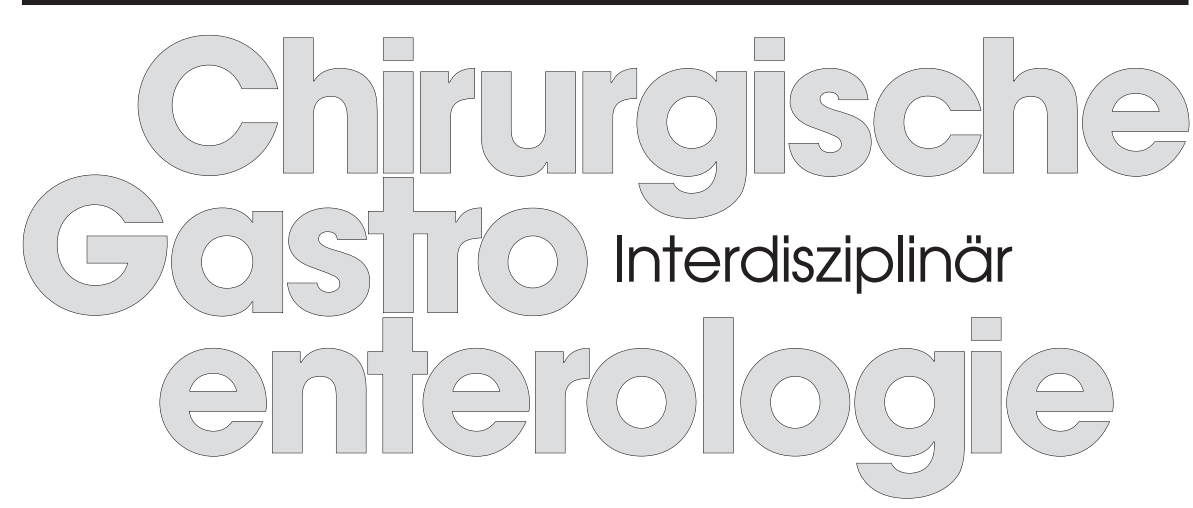

Hauptthema

C.-D. Heidecke,

M.M. Lerch, Greifswald (Hrsg.)

\title{
Komplikationen und Komplikationsmanagement im Gastrointestinaltrakt
}

Eingeladene Beiträge

Weitere Rubriken

Zertifizierte medizinische Fortbildung

Originalarbeiten

Fallbericht

Tagungen und Kongresse 
Band 24, Heft 2, Juni 2008

Nachruf

82 Steven Karger (1959-2008)

Editorial

83 Komplikationen und Komplikationsmanagement im Gastrointestinaltrakt

Heidecke, C.-D.; Lerch, M.M. (Greifswald)

Hauptthema

84 Komplikationsmanagement nach Ösophagektomie Westerholt, A.; Maier, S. (Greifswald); Bartels, H. (München); Heidecke, C.-D.; Glitsch, A. (Greifswald)

92 Management postoperativer Komplikationen nach Magenresektionen

Sendler, A.; Bergmann, U.; Henning, B.; Jonas, S. (Herne)

99 Endoskopische Komplikationen und endoskopisches Komplikationsmanagement an Kardia und Magen Kraft, M.; Mayerle, J.; Simon, P.; Aghdassi, A.; Lerch, M.M. (Greifswald)

103 Ätiopathogenese und Prävention der iatrogenen Pankreatitis

Ringel, J.; Wahnschaffe, U.; Lerch, M.M. (Greifswald)

108 Management postoperativer

Pankreaskomplikationen

Kutup, A.; Yekebas, E.F.; Izbicki, J.R. (Hamburg)

115 Management postoperativer und postinterventioneller Gallenwegskomplikationen Mihaljevic, A.L.; Kleeff, J.; Friess, H. (München)

124 Komplikationsmanagement nach Leberchirurgie und Lebertransplantation

Brockmann, J.; Vogel, T.; Senninger, N. (Münster)

134 Komplikationsmanagement von Leckagen nach extraperitonealen rektalen Anastomosen

Glitsch, A.; Schreiber, A.; Seltrecht, U.; Partecke, I.; Paul, H.; Patrzyk, M.; Heidecke, C.-D.; von Bernsdorff, W. (Greifswald)

142 Management postoperativer Komplikationen nach Kolonresektionen

Kujath, P.; Hofmann, M.; Shekarriz, H. (Lübeck)
Vol. 24, Issue 2, June 2008

Obituary

82 Steven Karger (1959-2008)

Editorial

83 Complications and Complication Management in the Gastrointestinal Tract

Heidecke, C.-D.; Lerch, M.M. (Greifswald)

Main Topic

84 Therapy of Complications Following Esophagectomy Westerholt, A.; Maier, S. (Greifswald); Bartels, H. (München); Heidecke, C.-D.; Glitsch, A. (Greifswald)

92 Management of Postoperative Complications after Gastric Resections

Sendler, A.; Bergmann, U.; Henning, B.; Jonas, S. (Herne)

99 Endoscopic Complications and Endoscopic Complication Management in Cardia and Stomach Kraft, M.; Mayerle, J.; Simon, P.; Aghdassi, A.; Lerch, M.M. (Greifswald)

103 Etiopathogenesis and Prevention of Iatrogenic Pancreatitis

Ringel, J.; Wahnschaffe, U.; Lerch, M.M. (Greifswald)

108 Management of Postoperative Complications in Pancreatic Surgery

Kutup, A.; Yekebas, E.F.; Izbicki, J.R. (Hamburg)

115 Management of Postoperative and Postinterventional Bile Duct Complications Mihaljevic, A.L.; Kleeff, J;; Friess, H. (München)

124 Complication Management after Liver Surgery and Liver Transplantation

Brockmann, J.; Vogel, T.; Senninger, N. (Münster)

134 Management of Leakages and Complications Following Extraperitoneal Rectal Anastomoses Glitsch, A.; Schreiber, A.; Seltrecht, U.; Partecke, I.; Paul, H.; Patrzyk, M.; Heidecke, C.-D.; von Bernsdorff, W. (Greifswald)

142 Management of Postoperative Complications Following Colonic Resections Kujath, P.; Hofmann, M.; Shekarriz, H. (Lübeck)

\section{KARGER}

(C) 2008 S. Karger GmbH, Freiburg

Fax +49 7614520714

Accessible online at:

E-mail Information@Karger.de www.karger.com/cga

www.karger.com 


\section{Band 24, Heft 2, Juni 2008}

Zertifizierte medizinische Fortbildung

146 Stadiengerechte Diagnostik und Therapie der akuten Divertikulitis des Colon sigmoideum Schreiber, A.; Heidecke, C.-D.; Glitsch, A. (Greifswald)

Originalarbeiten

156 Diagnostik und Therapie der gastroösophagealen Refluxerkrankung - Ergebnisse einer deutschlandweiten Umfrage Hartmann, J.; Nocon, M.; Naumann, H.; Hartmann, H.; Ordemann, J. (Berlin); Jacobi, C.A. (Wesseling)

162 Ergebnisse der Anwendung von Ethylpyruvat in einem experimentellen Kolitismodell

Karabeyoğlu,Ş.M.; Bozkurt, B.; Ünal, B.; Yilmaz, O.C.; Bilgihan, A.; Öz Atasay, F.; Üstün, H.; Cengiz, Ö. (Ankara)

167 Offenes versus laparoskopisches Management einer gastroduodenalen Ulkusperforation: Eine vergleichende monozentrische Erfahrung Li, B.; Yu, H.; Liu, J.; Yu, W.; Wang, F; Chen, B.; Hu, S. (Jinan)

\section{Fallbericht}

171 Isoliertes myeloides Sarkom des Dünndarms bei einem aleukämischen Patienten: Fallbericht und Literaturübersicht

Venizelos, I.D.; Frakandreas, G.; Tatsiou, Z.A.; Goulis, I. (Thessaloniki)

176 Innovationen

181 News / Ticker

182 Tagungen und Kongresse

184 Hinweise für Autoren

78 Impressum
Vol. 24, Issue 2, June 2008

Continuing Medical Education

146 Stadium-Adjusted Diagnostics and Treatment of Acute Diverticulitis of the Colon sigmoideum Schreiber, A.; Heidecke, C.-D.; Glitsch, A. (Greifswald)

Original Articles

156 Diagnosis and Therapy of Gastroesophageal Reflux - Results of a German Nationwide Survey Hartmann, J.; Nocon, M.; Naumann, H.; Hartmann, H.; Ordemann, J. (Berlin); Jacobi, C.A. (Wesseling)

162 Results of Ethyl Pyruvate Application in an Experimental Colitis Model

Karabeyoğlu, Ş.M.; Bozkurt, B.; Ünal, B.; Yilmaz, O.C.; Bilgihan, A.; Öz Atasay, F.; Üstün, H.; Cengiz, Ö. (Ankara)

167 Open versus Laparoscopic Management of Gastroduodenal Ulcer Perforation: A SingleInstitution Comparative Experience Li, B.; Yu, H.; Liu, J.; Yu, W.; Wang, F.; Chen, B.; Hu, S. (Jinan)

Case Report

171 Isolated Myeloid Sarcoma of the Small Intestine in an Aleukemic Patient - Case Report and Review of the Literature

Venizelos, I.D.; Frakandreas, G.; Tatsiou, Z.A.; Goulis, I. (Thessaloniki)

176 Innovations

181 News / Ticker

182 Meetings and Conferences

184 Guidelines for Authors

78 Imprint 